\title{
A Medical Error: To Disclose or Not to Disclose
}

Lubna Ghazal, Zulekha Saleem* and Gulzar Amlani

Aga Khan Hospital, Karachi, Sind, Pakistan

\begin{abstract}
Human error can occur in any profession. Medical errors are most commonly occurring errors in a health care system, which are responsible to delay patient's recovery and produce harm to patient. However, being as a health care professional, it is the requirement of professional code of ethics to do well and not to harm our patients. Historically, many of these errors were not disclosed to patients but the trend is emerging for more open disclosure of medical errors to patients and their families. The aim of this paper is to explain medical error and analyze this concept in the light of ethical principles. The paper will discuss the role of medical professionals in the disclosure of medical errors integrating an explicit ethical decision making process named MORAL.
\end{abstract}

\section{Introduction}

Medical error is defined as errors or mistakes committed by health professionals which result in harm to the patient [1]. They include errors in diagnosis (diagnostic errors), errors in the administration of drugs and other medications (medication errors), errors in the performance of surgical procedures, in the use of other types of therapy, in the use of equipment, and in the interpretation of laboratory findings [2]. It is also defined as the failure of a planned action to be completed as intended (i.e. error of execution) or the use of a wrong plan to achieve an aim (i.e. error of planning) [3]. In a broader sense patients value clear communication and responsiveness and if one lacks those, patients may perceive this as a medical error [4]. Generally, medical errors are considered to be as failed processes which may not essentially result in harm to a patient. Sometimes these medical errors may lead to an adverse event and at other times a near-miss.

Medical errors can be categorized in several ways; these categories include judgmental error, technical errors, expectation errors, and mechanical and system errors [1]. Errors can also be classified as skill-based, rule-based, and knowledge-based. Another mode of classification could be negligent and non-negligent error.

\section{Process of Reporting Medical Error; Disclosure versus Non-disclosure}

When an error or mistake occurs, the most common dilemma, faced by doctors and nurses, is whether to disclose or not to disclose the error to the patient. Research findings reveal that patients are keen to know about any error that caused them harm. The patient's bill of rights also demands to have full disclosure of an error. Several studies report that patients do verbalize that such disclosure would enhance their trust in their physicians' honesty and would reassure them that they are receiving complete information about their overall care. In addition, it was interesting to note that patients believed that human are not perfect they might do errors and "human nature" might lead health care workers to hide errors from patients [5].

\section{Principle of Beneficence and Non-maleficence}

The principle of beneficence in medical practice refers to avoid and prevent error by doing well [6]. The principle of non-maleficence emphasizes that one should not cause harm to oneself and others [7]. When patients come to a health care system to seek care, they trust the system and health care providers. They expect competency and believe that physician will provide the best treatment in accordance with the principle of beneficence. As a moral obligation, the principle of beneficence guides us to remove condition that will harm others, and prevent harm from occurring to others [7]. Disclosure of error to the patient will enhance the trust in physician and prevent law suit on to the hospital. Along with this, disclosure to the hospital management will help to improve processes and reduce errors for the future. Therefore, in the light of beneficence and non-maleficence, disclosure of error to patient and management is justified as a better option.

\section{Autonomy}

Autonomy is defined as the ability of an individual to make an informed, un-coerced and rational decision. [7]. According to the principle of autonomy it is patient's right to have full information about the treatment and any error if occurred. Physicians tend to hide the error when patient do not ask about it, and this is referred as deceptive approach $[5,8]$. Principle of autonomy supports truth telling therefore, disclosure of fact with the patient is highly justified.

\section{Kantian Theory}

Deontology or obligation based theory given by Kant is very important for physicians and nurses in the light of biomedical ethics. Kant asserts that ethics without obligation is no ethics and thus he emphasized on absolute duties or actions [7]. This theory implies doing well to people, telling truth, do no harm and respect autonomy of individual. Virtue ethics also support these principles. According to Kantian theory physicians have to follow standards of best care which are designed to protect patients $[7,8]$.

The physician patient relationship exists between individuals, not between a person and a "system", and telling injured patients the truth involves honest conversations between patients and the caregivers they know and trust [9]. According to Kant, lie is morally unacceptable and it is a strict obligation of health care professional to reveal facts with

*Corresponding author: Zulekha Saleem, Aga Khan Hospital, Karachi, Sind, Pakistan, Tel: 92 21-34930051; E-mail: zulekha.saleem@aku.edu

Received November 01, 2013; Accepted February 02, 2014; Published February 08,2014

Citation: Ghazal L, Saleem Z, Amlani G (2014) A Medical Error: To Disclose or Not to Disclose. J Clin Res Bioeth 5: 174. doi:10.4172/2155-9627.1000174

Copyright: $\odot 2014$ Ghazal L, et al. This is an open-access article distributed under the terms of the Creative Commons Attribution License, which permits unrestricted use, distribution, and reproduction in any medium, provided the original author and source are credited. 
the patient [7]. Therefore, based on Kantian theory discourse is again justified as the best option.

In the light of the above mentioned theories, health care professional should strive for more truth telling and disclosure of medical errors. Disclosure will enhance patient's trust on physician and the health care system. The study showed disclosure of errors has reduced the chances of lawsuits cases on hospital or on physician [10]. Physician also supports the disclosure of error against the choice of no disclosure. On the contrary, some of the physician disagrees to disclose complete information about the error to patient. Some of the physician also believed that if patient do not enquire about information there is no need to tell $[3,5,7]$. However, this is an ethical dilemma and it should be resolved by adequate ethical frameworks and models.

\section{"MORAL" A Step By Step Process to Help in Ethical Decision Making}

The ethical theories and reasoning do not solve ethical dilemma rather provide a direction for action. To address a medical error, the health care provider should handle the situation and correct the error; secondly, the error should be disclosed $[11,12]$. The MORAL is an extensive and clear ethical decision making model based on five steps of ethical decision making process proposed by Patricia Crisham in 1985. In MORAL, The " $M$ " stands for Massage the Dilemma, "O" stands for outlines options, " $R$ " review criteria and resolve, "A" stands for affirm position and act, and "L" stands for look back [10].

The first step of MORAL Model involve massage the dilemma; this includes data collection about situation, people, and their value in conflict [10]. This means that a person should analyze the context in which error occurred and should identify the key aspects, facts, underlying ethical principles and values, etc. In the second step one has to identify the possible options for rectification of the error. Then, one should undergo constructive thinking and reflection to analyze the pros and cons of each option. In the third step one has to identify the moral criteria to select the appropriate course of actions, based on moral judgment [10]. On the basis of moral judgment with the help of ethical principles and theories, one has to select the best action in patient's interest. In the forth step, one should practically integrate the selected option in the patients' scenario to resolve the dilemma keeping in view the ethical principles. The last and final step in MORAL emphasis on evaluating the ethical dilemma and its resolution and implementation with final course of appropriate strategies [5]. Evaluation help us to see the effectiveness of our decision of choosing best option in patient's interest.

\section{Role of Health Care Providers in the Disclosure of Medical Error}

As a health care provider, one encounters a number of medical errors every day. The literature strongly suggests that errors should be disclosed to the patients. Yet it appears this practice is uncommon in health care. Health care team feels upset and guilty when medical errors occur. Health care providers involved in an error also report disappointment for their failure to be safe and competent. They also feel anxious about the error's repercussion on their professional growth. In addition, they also report fearful about a possible lawsuit against them or to the hospital in which they are practicing [11-13].

This contradicts the health care providers professional responsibility to give the high quality of medical care and to act in the patient's best interest. Therefore, the health care providers should be taught about the underpinnings of ethical principles that support truth telling and honesty. Moreover, they should reflect on the patient's expectations concerning disclosure and the factors that hinder disclosure so that the gap between theory and practice can be bridged. Along with that, they should be encouraged to integrate the ethical decision making framework like MORAL on the clinical. In addition, they should be taught about the specific communication techniques and pertinent apologizing words to be used while disclosing the medical error. Along with that a non-penalizing error reporting culture should be created so that health care providers do not feel threatened. At the same time, the causes and effects of medical error could be investigated and appropriate actions should be initiated so that one learns from their mistakes and prevent it in future clinical practice.

\section{Conclusion}

In conclusion, health care providers encounter different medical error in their day to day practices. The disclosure of a medical error is an ethical dilemma that requires deliberative thinking and reflection by the health care providers. It is suggested that disclosure of medical error should be encouraged keeping in view the principle of beneficence, non-maleficence, patient's autonomy and Kant's theory. This disclosure will lead to better satisfaction from the patients and health care providers. Hence, medical error should be addressed and reported as this can help to improve system and prevent such errors in future and will contribute for more professional growth.

\section{Key Message}

In my view, it is indeed a challenge to reveal the medical error and there are diverse factors that affect a physician's/nurse or health care provider's readiness to disclose their errors. Many times an error is not specifically an individual person's fault but a team or system-based problem. However, disclosure of medical errors can be a very individual issue because sometimes only one person knows about it. Beyond our job duty to the patient or the hospital, we have a responsibility to ourselves to practice responsible and honest medicine.

"Remember, there is nothing you can do to change the past but you can use its lessons to improve your future." Rabbi Abraham J Twerski, MD

\section{References}

1. Krizek TJ (2000) Surgical error: ethical issues of adverse events. Arch Surg 135: 1359-1366.

2. Dictionary MW (2003) Merriam-Webster, Incorporated.

3. Bates DW, Cohen M, Leape LL, Overhage JM, Shabot MM, et al. (2001) Reducing the frequency of errors in medicine using information technology. $J$ Am Med Inform Assoc 8: 299-308.

4. Joint Commission Journal on Quality and Patient Safety (2007) Study Indicates that Patients' Broad definition of medical error can Undermine Satisfaction with Care. Journal of Radiology Nursing Pages 35-36.

5. Gallagher TH, Bell SK, Smith KM, Mello MM, McDonald TB (2009) Disclosing harmful medical errors to patients: tackling three tough cases. Chest 136: 897903.

6. Crigger NJ (2004) Always having to say you're sorry: an ethical response to making mistakes in professional practice. Nurs Ethics 11: 568-576.

7. Beauchamp TL, Childress JF (2001) Principles of Biomedical Ethics. (5thedn) Oxford, New York, USA.

8. Heubel F, Biller-Andorno N (2005) The contribution of Kantian moral theory to contemporary medical ethics: a critical analysis. Med Health Care Philos 8: 5-18. 
Citation: Ghazal L, Saleem Z, Amlani G (2014) A Medical Error: To Disclose or Not to Disclose. J Clin Res Bioeth 5: 174. doi:10.4172/21559627.1000174

Page 3 of 3

9. Berlinger N, Wu AW (2005) Subtracting insult from injury: addressing cultural expectations in the disclosure of medical error. J Med Ethics 31: 106-108.

10. Crisham $P$ (1985) Moral: how can I do what's right? Nurs Manage 16: 42A-42N.

11. Seiden SC, Barach $P$ (2006) Wrong-side/wrong-site, wrong-procedure, and wrong-patient adverse events: Are they preventable? Arch Surg 141: 931-939.
12. Rosner F, Berger JT, Kark P, Potash J, Bennett AJ (2000) Disclosure and prevention of medical errors. Committee on Bioethical Issues of the Medical Society of the State of New York. Arch Intern Med 160: 2089-2092.

13. Lamb RM, Studdert DM, Bohmer RM, Berwick DM, Brennan TA (2003) Hospital disclosure practices: results of a national survey. Health Aff (Millwood) 22: 7383. 